Draft VERSion OCTOBER 3, 2016

Preprint typeset using IATEX style AASTeX6 v. 1.0

\title{
HIGH ENERGY PROPERTIES OF THE FLAT SPECTRUM RADIO QUASAR 4C 50.11
}

\author{
Jianeng Zhou, V. Jithesh, Chen Liang and Zhongxiang Wang \\ Shanghai Astronomical Observatory, Chinese Academy of Sciences, 80 Nandan Road, Shanghai 200030, China
}

\begin{abstract}
We investigate the $\gamma$-ray and X-ray properties of the flat spectrum radio quasar (FSRQ) $4 \mathrm{C} 50.11$ at redshift $z=1.517$. The Fermi Large Area Telescope (LAT) data indicate that this source was in an active state since 2013 July. During the active period, the $\gamma$-ray flux increased by more than a factor of three and two distinct flares were detected with the variability timescale as short as several hours. The $\gamma$-ray spectra can be well fitted by a log-parabola. From the fitting, we find a correlation between the peak energy and spectral curvature for the $\gamma$-ray spectra, which is the first time seen in $\gamma$-ray emission from a blazar. The Swift X-ray Telescope (XRT) data show that the source was variable at X-ray energies, but no evidence shows flux or spectral changes related to the $\gamma$-ray activity. The broad-band X-ray spectrum obtained with Swift XRT and NuSTAR is well described by a broken PL model, with an extremely hard spectrum $\left(\Gamma_{1} \sim 0.1\right)$ below the break energy, $E_{\text {break }} \sim 2.1 \mathrm{keV}$, and $\Gamma_{2} \sim 1.5$ above the break energy. The spectral steepening below $\sim 3 \mathrm{keV}$ is likely due to the low energy cut-off in the energy distribution of the photon-emitting electron population. Both the $\gamma$-ray and X-ray emission appear harder when brighter. The broad-band spectral energy distribution (SED) is constructed for the source, and we provide a model fit to the SED. Our modeling suggests that the emission region should be outside the broad line region, and the properties of the region indicate a jet with $42 \%$ of the Eddington power during the active state.
\end{abstract}

Keywords: galaxies: jets — gamma rays: galaxies — quasars: individual (4C 50.11) — radiation mechanisms: non-thermal

\section{INTRODUCTION}

Blazars are radio-loud active galactic nuclei (AGNs) with relativistic jets pointing towards the Earth (Urry, C. M. \& Padovani, P. 1995). Because of the Doppler beaming effect, emission from a jet dominates the broad-band spectral energy distribution (SED) from radio to $\gamma$-rays energies. The SEDs usually have two broad bumps in a $\log \nu-\log \nu f_{\nu}$ diagram. While the lowenergy bump usually peaks from infrared to X-ray energies, which are believed to be the synchrotron emission of non-thermal electrons, the high-energy bump peaks from X-ray to $\gamma$-ray bands, which is considered to be the inverse Compton (IC) emission of the same electron population. For the IC emission, the seed photons can come from the low-energy synchrotron emission, broad line region (BLR), or dusty torus. Because of the synchrotron self-absorption effect, blazars tend to have flat radio spectra with spectral index $\alpha<0.5$. As a subclass of blazars, flat spectrum radio quasars (FSRQs) have strong optical emission lines (equivalent width $>5 \AA$ ), comparing to BL Lac objects that show no or very weak emission lines.

In the current third Fermi Large Area Telescope (LAT) source catalog (3FGL), the dominant extragalactic $\gamma$-ray sources are blazars (Acero et al. 2015). These blazars show violent variability, especially at high energy bands. The minimal variable timescale detected with Fermi-LAT has reached less than half an hour (e.g. PKS 1510-089, Foschini 2013) and the variation amplitude can be two orders of magnitude (e.g., 3C 454.3, Abdo et al. 2011). Detailed studies of spectra and variabilities are essential for determining the location and mechanism of radiation from the jets of the blazars.

The FSRQ 4C 50.11 (also known as NRAO 150) is one of the strongest radio and millimeter AGN sources in the northern sky (Pauliny-Toth et al. 1966; Agudo et al. 2008, 2010). The VLBI monitoring observations showed that the inner jet (inner 0.5 mas from the core) exhibits superluminal motions with apparent speed $v_{a p p} \approx 3.3 \mathrm{c}$ and a large, $>100^{\circ}$ projected misalignment of the jet within the inner 0.5 mas to 1 mas from the core. These properties imply that a relativistic jet points toward the Earth with a very small viewing angle (Agudo et al. 2007). Acosta-Pulido et al. (2010) measured the redshift using near-IR spectroscopic data (exhibiting strong $\mathrm{H} \alpha$ and $\mathrm{H} \beta$ emission lines), and derived the cosmological redshift $z=1.517 \pm 0.002$, which corresponds to the luminosity distance $d_{L}=11.2 \times 10^{3} \mathrm{Mpc}$. They also predicted that 4C 50.11 would be a high-luminosity $\gamma$-ray emitter and one of the most powerful blazars. Foschini (2010) reported the detection of $\gamma$-ray emission from $4 \mathrm{C}$ 50.11 with LAT on board the Fermi satellite. Using almost 20 months of data, he provided the $\gamma$-ray flux above $100 \mathrm{MeV}, F_{100 \mathrm{MeV}}=3.2 \pm 1.1 \times 10^{-8}$ photons $\mathrm{cm}^{-2} \mathrm{~s}^{-1}$, 
and photon index $\Gamma=2.6 \pm 0.2$. After the $\gamma$-ray flaring activity around $\sim$ MJD 56686 (2014 January 29), Swift target-of-opportunity observations were performed (Carpenter et al. 2014; Krauss et al. 2014).

For the purpose of fully studying this high-energy source, we collected its Fermi-LAT and available X-ray data, which include 15 Swift observations and one $N u S$ $T A R$ observation, and performed detailed analysis of the data. In this paper, we present the results from our analysis. In the following, $\S 2$ describes the analysis of the Fermi-LAT data and provides the results. The Swift and NuSTAR observations and data-analysis results are presented in $\S 3$ and $\S 4$ respectively. We discuss the overall properties of the source in $\S 5$. In $\S 6$, we summarize our results.

\section{FERMI-LAT DATA : ANALYSIS AND RESULTS}

\subsection{Data and Source Determination}

We used approximately seven-years Fermi-LAT Pass 8 data in this work, which are from MJD 54682 (2008-08$04)$ to MJD 57252 (2015-11-26), with the energy range from $100 \mathrm{MeV}$ to $100 \mathrm{GeV}$. During the time period, 4C 50.11 was in an active state from MJD 56482 (2013-0709) to the end of the data. The Fermi Science Tools v10r0p5 package was used to analyze the data, with the P8R2_SOURCE_V6 instrument response functions (IRFs) applied. To avoid contamination from $\gamma$-rays reflected by the Earth, we selected the events with zenith angles $\leq 90^{\circ}$.

In the analysis, photons from a $20^{\circ} \times 20^{\circ}$ square region of interest (ROI) centered at the position of $4 \mathrm{C}$ 50.11 were selected, and binned into spatial pixels of $0.1^{\circ} \times 0.1^{\circ}$. The first run of the analysis, using gtlike, was performed with the binned likelihood method to derive the sky map model. We modeled the events considering the components of the target and background. The background was composed of sources in the 3FGL catalog (Acero et al. 2015) within the ROI and diffuse components. The latter included the Galactic diffuse model (gll_iem_v06.fits) and isotropic background (iso_P8R2_SOURCE_V6_v06.txt).

To confirm the spatial association of $\gamma$-ray emission with $4 \mathrm{C} 50.11$, we calculated a $2^{\circ} \times 2^{\circ}$ Test Statistic (TS) map centered at its coordinates. A putative point source was assumed and moved through a grid of locations on the sky by maximizing - $\log$ (likelihood) at each grid point. In this step, the target source that corresponds to $4 \mathrm{C} 50.11$ was unmodeled (i.e., removed from the model file). All parameters of point sources in the ROI, except the diffuse components, were fixed at the 3FGL catalog values. To reduce the contamination due to the large point spread function (PSF) at low energies, we only used photons above $1 \mathrm{GeV}$ for TS map calculation. The $\gamma$-ray source was significantly detected, with a maximum TS value of $\simeq 280$. We derived the position of the source, and the best-fit position is $\mathrm{RA}=59.872^{\circ}$, Decl. $=50.968^{\circ}(\mathrm{J} 2000.0)$ with a positional uncertainty of $0.022^{\circ}$. The derived position has an offset of $1.9^{\prime}$ from
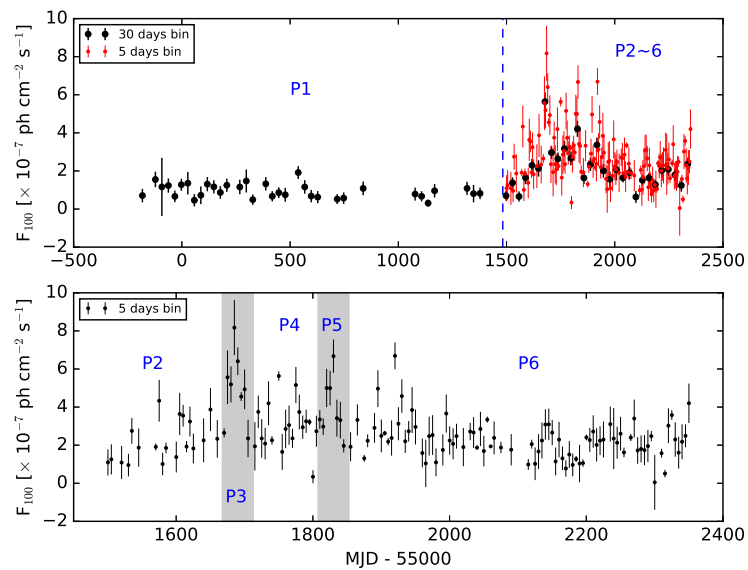

Figure 1. Fermi-LAT light curves of 4C 50.11. Different time intervals are defined based on the light curve properties. Upper: Long-term light curves binned with 30 days (black points) and 5 days (red points). Lower: Light curve of $4 \mathrm{C}$ 50.11 during the $\gamma$-ray active period from MJD 56482, binned with 5 days.

the radio position of $4 \mathrm{C} 50.11$ (Fey et al. 2004), consistent within $2 \sigma$ error circle of the Fermi position. No other known high-energy sources are within the error circle.

\subsection{Light Curve Analysis}

Since 4C 50.11 was active from $\sim$ MJD 56482 , showing $\gamma$-ray flares, a 5 -days binned light curve was derived using the binned likelihood method. Normalizations of all point sources within $5^{\circ}$ from the target and sources with variable index ${ }^{1} \geq 72.44$ were set free. For the purpose of studying the flaring variability in detail, 24-, 12-, and 6 -hours binned light curves were also created. For these light curves, an unbinned likelihood method was used due to the low statistics, and only the normalizations of variable sources were set free in the background model.

Figure 1 shows the $\gamma$-ray light curves of $4 \mathrm{C} 50.11$ in 0.1-100 GeV, with the upper panel covering the entire Fermi observation time period (binned with 30 days) and the lower panel covering the $\gamma$-ray active period starting from $\sim$ MJD 54682. Six time intervals are defined based on the light curves (see Figure 1): P1 is the time period before the active state and $\mathrm{P} 2-\mathrm{P} 6$ the time periods covering the active state, which lasts for more than two years. There are two distinct $\gamma$-ray flares in the active state, P3 and P5. The timescale of turning to be flaring was less than one day.

We investigated the active state of $4 \mathrm{C} 50.11$ in different energy bands. Two flaring events (P3 and P5) are clearly seen in the $0.1-1 \mathrm{GeV}$ energy band (Figure 2). However in the $>1 \mathrm{GeV}$ energy light curve, the flares are not significant comparing to the rest part of the light

\footnotetext{
${ }^{1}$ http://fermi.gsfc.nasa.gov/ssc/data/access/lat/4yr_catalog/
} 


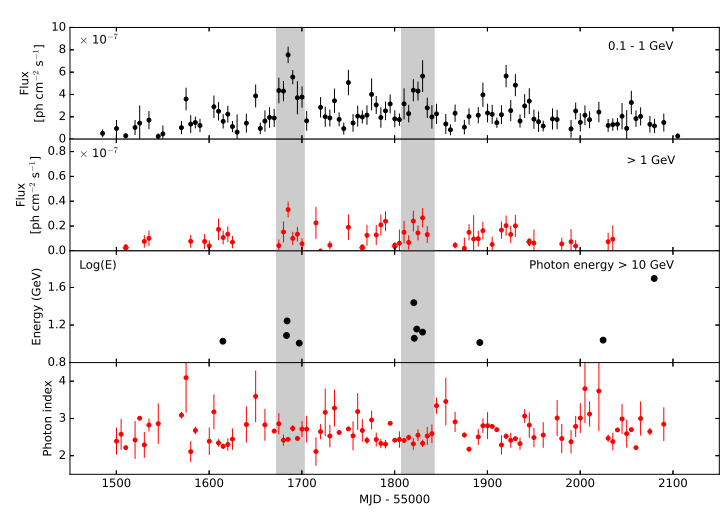

Figure 2. Light curves of $4 \mathrm{C} 50.11$ binned with 5 days during the $\gamma$-ray active period. From top to bottom: $0.1-1 \mathrm{GeV}, 1-$ $100 \mathrm{GeV}$, arriving times of photons with energy $>10 \mathrm{GeV}$, and photon index from single PL fitting.

Table 1. List of energy $>10 \mathrm{GeV}$ photons from the direction of $4 \mathrm{C} 50.11$

\begin{tabular}{ccccc}
\hline \hline Time & Energy & \multicolumn{3}{c}{ Probability (\%) } \\
\cline { 3 - 5 }$(\mathrm{MJD})$ & $(\mathrm{GeV})$ & $4 \mathrm{C} 50.11$ & Galactic BG & isotropic BG \\
\hline 56614.842 & 10.7 & 11.4 & 75.2 & 13.4 \\
56683.446 & 12.3 & 38.0 & 51.3 & 10.7 \\
56684.055 & 17.5 & 1.2 & 79.9 & 18.9 \\
56697.165 & 10.2 & 9.2 & 77.6 & 13.2 \\
56820.629 & 27.4 & 2.9 & 72.5 & 24.6 \\
56821.168 & 11.5 & 2.7 & 82.4 & 14.9 \\
56823.813 & 14.4 & 1.4 & 78.8 & 19.8 \\
56830.112 & 13.3 & 8.7 & 73.8 & 17.5 \\
56891.826 & 10.3 & 86.9 & 11.2 & 1.9 \\
57024.744 & 10.9 & 2.0 & 83.8 & 14.2 \\
57079.817 & 49.7 & 3.0 & 70.6 & 26.4 \\
\hline
\end{tabular}

Note - Column 3-5 present the probability of each photon belong to 4C 50.11, Galactic interstellar emission background and isotropic extragalactic emission background.

curve, and the overall photon flux is an order of magnitude lower than that in the $0.1-1 \mathrm{GeV}$ energy band. In addition, we found eleven $>10 \mathrm{GeV}$ photons during the active period, of which seven are within the time intervals of the flares (see Table 1 and Figure 2). We checked their probabilities of coming from $4 \mathrm{C} 50.11$, using gtsrcprob. Although the probabilities are generally not high, we note that the time length of P3 and P5 is only $10 \%$ of that from P2 to P6, showing that the highenergy photons were likely related to the flares. In any case, one $10.3 \mathrm{GeV}$ photon has a high value of $\sim 87 \%$.

We modeled the photons in each time bin with a single power law (PL) model (see below for detailed discussion about spectral modeling in Section 2.3). As shown in the bottom panel of Figure 2, the derived photon index varies with time and flux. A possible property during the flares is that the photon index hardened (i.e., when the flux was high).
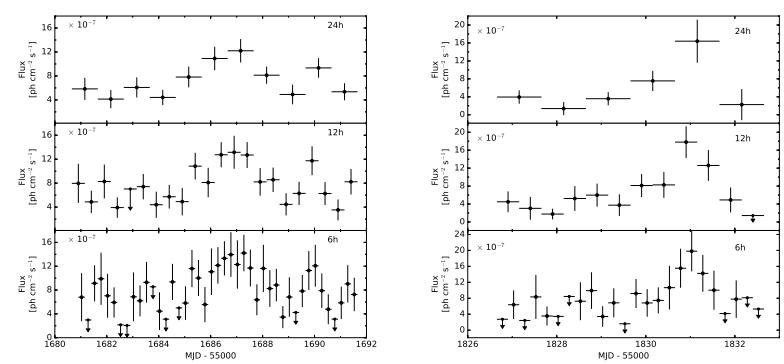

Figure 3. Light curves of two flares, P3 (left) and P5 (right). From top to bottom: the light curves binned with 24,12 , and 6 hours, respectively.

Details of the two flares are shown in Figure 3. The first one started at $\sim$ MJD 56685 and lasted for $\sim 5$ days. During this period, the flare has a relatively flat profile, while a small sub-flare around MJD 56690 is seen, which lasted only $\sim 2$ days. We extracted the spectrum of the first flare and fitted it with a single PL model. From fitting, we found averaged photon flux $F_{100 \mathrm{MeV}}=6.3 \pm 0.3 \times 10^{-7}$ photons $\mathrm{cm}^{-2} \mathrm{~s}^{-1}$ and photon index $\Gamma=2.55 \pm 0.04$. The second flare, with the peak around $\sim$ MJD 56831, has a different shape compared to the first one. The averaged photon flux of this flare was $F_{100 M e V}=4.6 \pm 0.3 \times 10^{-7}$ photons $\mathrm{cm}^{-2} \mathrm{~s}^{-1}$ and photon index $\Gamma=2.44 \pm 0.05$. The peak flux (given from the 6-hours light curves) was $2.0 \pm 0.5 \times$ $10^{-6}$ photons $\mathrm{cm}^{-2} \mathrm{~s}^{-1}$.

\subsection{Spectral Analysis}

We analyzed the $\gamma$-ray spectra of $4 \mathrm{C} 50.11$ in the different time intervals (P1-P6) and the total time interval of the combined P2-P6. Models of simple PL $(d N / d E \propto$ $E^{\Gamma}$ ), broken PL (BPL; $d N / d E \propto E^{-\Gamma_{1}}$ for $E<E_{\text {break }}$, and $d N / d E \propto E^{-\Gamma_{2}}$ for $\left.E>E_{\text {break }}\right)$, and log-parabola $\left(d N / d E \propto\left(E / E_{b}\right)^{-\alpha-\beta \log \left(E / E_{b}\right)}\right)$ were considered. The results are given in Table 2. Based on the likelihood comparisons, the latter two models are slightly more favored over the PL.

In the log-parabola model, the peak energy in $\log \nu-$ $\log \nu L \nu$ can be expressed as $\log E_{p}=\log E_{b}+(2-\alpha) / 2 \beta$ and the curvature is represented by $1 / \beta$ (Chen 2014). For the spectra of the time intervals of $\mathrm{P} 1-\mathrm{P} 6$ and that of the combined $\mathrm{P} 2-\mathrm{P} 6$, we calculated the peak energies and curvatures. The results are shown in Figure 4, in which because $\beta=0$ during $\mathrm{P} 1$, the data point is not included. A correlation between the peak energy and curvature is apparent. We performed the Pearson test and obtained correlation coefficient $r=-0.94$ and chance probability $p=0.0055$. Another correlation exists between the photon index and flux when the single PL model is considered. In Figure 5, the parameter values are shown. The Pearson test gave correlation coefficient $r=-0.87$ and chance probability $p=0.0080$.

\section{SWIFT DATA : ANALYSIS AND RESULTS}


Table 2. $\gamma$-ray spectral fitting results for $4 \mathrm{C} 50.11$ in different time intervals

\begin{tabular}{|c|c|c|c|c|c|c|c|}
\hline Interval & Model & $\alpha / \Gamma / \Gamma_{1}$ & $\bar{\beta} \beta / \Gamma_{2}$ & $\begin{array}{l}\mathrm{E}_{\text {break }} \\
(\mathrm{GeV}) \\
\end{array}$ & TS & $-2 \Delta \mathrm{L}$ & $\begin{array}{l}\text { Flux } \\
\left(\times 10^{-7}\right) \\
\end{array}$ \\
\hline \multirow{3}{*}{$\mathrm{P} 1$} & $\mathrm{PL}$ & $3.01(3)$ & $\ldots$ & $\ldots$ & 303 & $\ldots$ & $6.99(35) \mathrm{E}-1$ \\
\hline & $\log \mathrm{P}$ & $3.01(8)$ & $0.00(0)$ & $\ldots$ & 304 & 0.0 & $6.99(48) \mathrm{E}-1$ \\
\hline & $\mathrm{BPL}$ & $2.97(17)$ & $3.15(77)$ & $1.0(1)$ & 303 & 0.2 & $6.94(45) \mathrm{E}-1$ \\
\hline \multirow{3}{*}{$\mathrm{P} 2$} & $\mathrm{PL}$ & $2.63(7)$ & $\ldots$ & $\ldots$ & 234 & $\ldots$ & $1.65(15)$ \\
\hline & $\operatorname{LogP}$ & $2.23(13)$ & $0.18(6)$ & $\ldots$ & 236 & 6.4 & $1.50(15)$ \\
\hline & $\mathrm{BPL}$ & $2.38(11)$ & $3.30(43)$ & $1.0(3)$ & 237 & 6.8 & $1.52(16)$ \\
\hline \multirow{3}{*}{ P3 } & $\mathrm{PL}$ & $2.55(4)$ & $\ldots$ & $\ldots$ & 654 & $\ldots$ & $6.30(30)$ \\
\hline & $\log \mathrm{P}$ & $2.42(10)$ & $0.06(4)$ & $\ldots$ & 651 & 3.8 & $6.14(39)$ \\
\hline & $\mathrm{BPL}$ & $2.44(8)$ & $2.87(24)$ & $1.0(2)$ & 652 & 5.0 & $6.11(39)$ \\
\hline \multirow{3}{*}{$\mathrm{P} 4$} & $\mathrm{PL}$ & $2.61(6)$ & $\ldots$ & $\ldots$ & 347 & $\ldots$ & $2.87(21)$ \\
\hline & $\log \mathrm{P}$ & $2.17(13)$ & $0.22(6)$ & $\ldots$ & 304 & 10.6 & $2.63(21)$ \\
\hline & $\mathrm{BPL}$ & $2.37(9)$ & $3.40(47)$ & $1.0(3)$ & 303 & 9.2 & $2.69(22)$ \\
\hline \multirow{3}{*}{$\mathrm{P} 5$} & $\mathrm{PL}$ & $2.44(5)$ & $\ldots$ & $\ldots$ & 358 & $\ldots$ & $4.59(28)$ \\
\hline & $\log \mathrm{P}$ & $2.22(8)$ & $0.10(3)$ & $\ldots$ & 359 & 2.4 & $4.37(29)$ \\
\hline & $\mathrm{BPL}$ & $2.26(7)$ & $2.90(22)$ & $1.0(0)$ & 361 & 3.8 & $4.35(34)$ \\
\hline \multirow{3}{*}{$\mathrm{P} 6$} & $\mathrm{PL}$ & $2.69(5)$ & $\ldots$ & $\ldots$ & 504 & $\ldots$ & $2.10(12)$ \\
\hline & $\log \mathrm{P}$ & $2.51(9)$ & $0.08(4)$ & $\ldots$ & 503 & 3.2 & $2.03(13)$ \\
\hline & $\mathrm{BPL}$ & $2.58(7)$ & $2.97(21)$ & $1.0(2)$ & 504 & 2.6 & $2.05(13)$ \\
\hline \multirow{3}{*}{$\mathrm{P} 2-\mathrm{P} 6$} & $\mathrm{PL}$ & $2.63(2)$ & $\ldots$ & $\ldots$ & 2175 & $\ldots$ & $2.38(7)$ \\
\hline & $\operatorname{LogP}$ & $2.35(5)$ & $0.14(2)$ & $\ldots$ & 2180 & 32.2 & $2.38(7)$ \\
\hline & $\mathrm{BPL}$ & $2.45(4)$ & $3.29(17)$ & $1.2(1)$ & 2191 & 36.4 & $2.26(7)$ \\
\hline
\end{tabular}

Note-Bracketed numerics are errors at values. Flux is in units of $\mathrm{ph} \mathrm{cm}^{-2} \mathrm{~s}^{-1}$. $-2 \Delta L$ indicates the difference of $\log$ (Likelihood) of the fit with respect to the single power law fit.

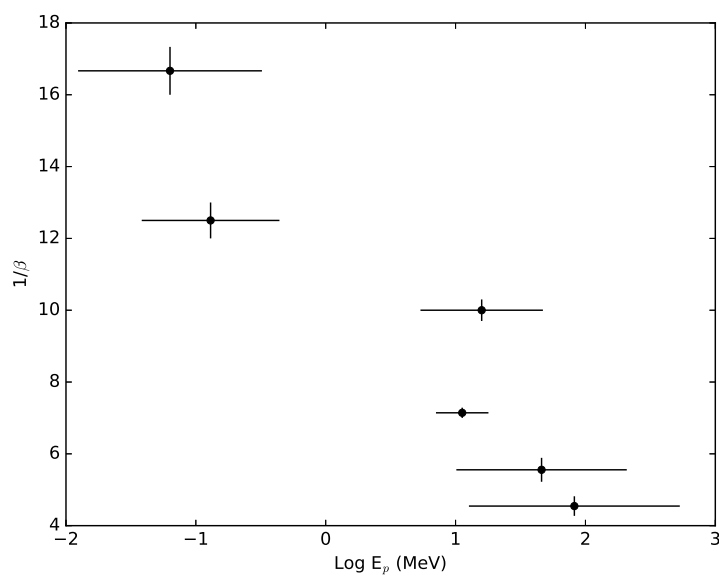

Figure 4. Spectral curvature versus peak energy for the spectra obtained in the different time intervals, when the spectra were fitted with a log-parabola model. For P1, because $\beta=0$, no data point is for the time interval.

\subsection{Swift XRT}

The Swift satellite (Gehrels et al. 2004) performed 15 observations of 4C 50.11 between 2007 January and 2015 December. We utilized archival data from the X-ray telescope (XRT; Burrows et al. 2005) on board Swift. The XRT data were processed with standard filtering and screening criteria, using the XRTPIPELINE version

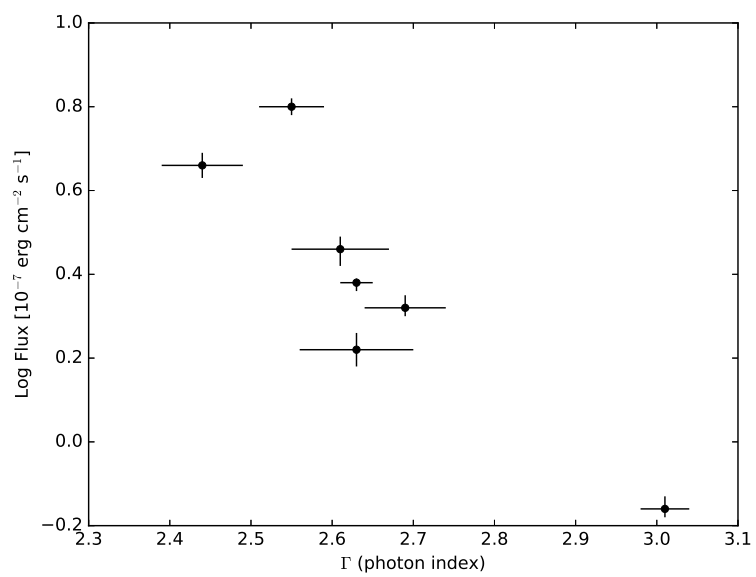

Figure 5. Flux versus photon index for the spectra obtained in the different time intervals, when they were fitted with a PL model.

0.13.0 in the HEASOFT package version 6.15.1. The photon-counting (PC) mode data were collected from all the observations. Since the source had low count rates $\left(<0.1\right.$ counts $\left.\mathrm{s}^{-1}\right)$, the pile-up correction was not required. Source events were extracted from a circular region with a radius of $47^{\prime \prime}$, while background events were extracted from a circular region of the same radius, with the standard grade filtering of $0-12$. We generated 


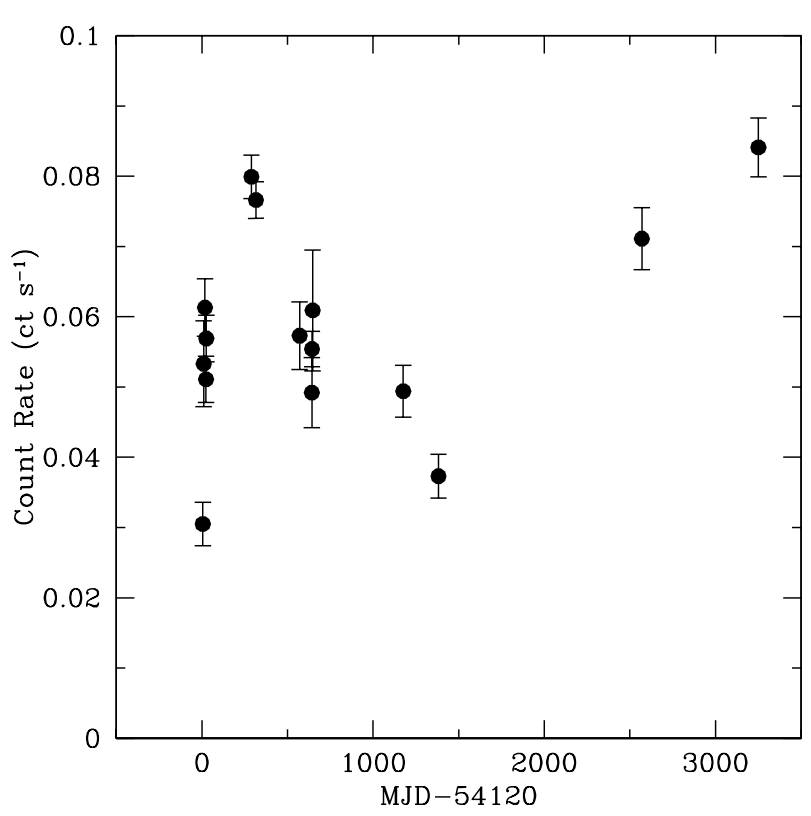

Figure 6. Count rate variations of $4 \mathrm{C} 50.11$ seen in the Swift XRT observations over year 2007-2015.

the ancillary response files with the tool XRTMKARF and used the spectral redistribution matrices available in the calibration data base (CALDB) version 20151105. The spectra were binned to contain at least 20 counts per bin, which allowed the $\chi^{2}$ spectral fitting. In other cases, where there were no sufficient spectral counts, the Cash Statistics (Cash 1979) was used for spectral modeling. We fitted the Swift XRT spectra with an absorbed PL model in the $0.3-10 \mathrm{keV}$ energy band. The absorption was incorporated by using the photoelectric absorption model tbabs (Wilms et al. 2000), which was fixed at the Galactic value, $6.93 \times 10^{21} \mathrm{~cm}^{-2}$ (Kalberla et al. 2005). The results are reported in Table 3. All errors are given at the $90 \%$ confidence level.

The obtained long-term Swift XRT count-rate curve of $4 \mathrm{C} 50.11$ is shown in Figure 6. During year 20072015 , the source showed variability with the count rates varying by a factor of $\sim 3$. We also observed an increase of the count rate in the latest Swift XRT observations and it reached the maximum value of $\sim 0.08$ counts $\mathrm{s}^{-1}$ on 2015 December 14.

For four observations, conducted on 2007 November 5 (Obs ID: 00036308001), 2007 December 2 (Obs ID: 00036308002), 2008 October 24 (Obs ID: 00036308005), and 2015 December 14 (Obs ID: 00080948001), there are enough spectral counts to test other spectral models. We initially added an extra absorption component at the redshift of the source (ztbabs) to the PL model. This combined model improved the spectral fit for three observations (with $\Delta \chi^{2} \sim 7-9$ for the loss of one degree of freedom at a confidence level of $>98 \%$ ) over the single PL. For the remaining one (Obs ID: 00036308005), the spectral fit was marginally improved $\left(\Delta \chi^{2} \sim 2\right)$ at a con- fidence of $\sim 86 \%$. The extra absorption at the redshift of the source $\left(\mathrm{N}_{\mathrm{H}}^{z}\right)$ is in a range of $6.5-12.6 \times 10^{22} \mathrm{~cm}^{-2}$ for these four observations.

Next, we attempted a broken PL model for all the four observations. For the 2007 November observation, the broken PL fit resulted in $\Gamma_{1}<0.99$ below the break energy $E_{\text {break }}=2.56_{-1.23}^{+0.58} \mathrm{keV}$ and $\Gamma_{2}=1.68_{-0.42}^{+0.36}$ above the break energy, $\left(\chi^{2} /\right.$ d.o.f $\left.=24.8 / 28\right)$. For the 2007 December observation, the broken PL provided an acceptable fit with $\Gamma_{1}=0.12_{-0.70}^{+0.58}, E_{\text {break }}=2.08_{-0.24}^{+0.61} \mathrm{keV}$, and $\Gamma_{2}=1.35_{-0.19}^{+0.20}\left(\chi^{2} /\right.$ d.o.f $\left.=38.9 / 38\right)$. The photon index $\Gamma_{1}=-0.31_{-1.20}^{+1.10}, E_{\text {break }}=2.18_{-0.25}^{+0.91} \mathrm{keV}$, and $\Gamma_{2}=1.80_{-0.33}^{+0.59}$ were obtained for the observation in 2015 $\left(\chi^{2} /\right.$ d.o.f $\left.=15.8 / 15\right)$. This model provided an improvement to the spectral fit for the three observations, with $\Delta \chi^{2} \sim 9-11$ for the loss of two extra degrees of freedom at a probability of $>98 \%$ (from F-test), over the single PL fit. The F-test results suggest that the broken $\mathrm{PL}$ is the best-fit spectral model for the source. However for the 2008 October observation, the broken PL model provided a marginally improved spectral fit (at a probability of $<80 \%$ ) over the single PL. Moreover, the break energy obtained $\left(E_{\text {break }} \sim 3.26 \mathrm{keV}\right)$ in this observation was not well constrained. We thus fixed $E_{\text {break }}$ at $3.26 \mathrm{keV}$, which yielded $\Gamma_{1}=0.85_{-0.31}^{+0.29}$ and $\Gamma_{2}=1.47_{-0.41}^{+0.44}\left(\chi^{2} /\right.$ d.o.f $\left.=16.1 / 22\right)$.

\subsection{Swift BAT}

The Swift Burst Alert Telescope (BAT; Barthelmy et al. 2005) observed the source with short-exposure observations, where the hard X-ray flux of the source was below the sensitivity of the BAT instrument. Therefore no BAT analysis was conducted here. However the source is included in the Swift BAT 70-month hard X-ray catalog (Baumgartner et al. 2013). The reported results of the hard X-ray spectrum (14-195 keV energy range) were a PL with photon index $\Gamma_{X}=1.51 \pm 0.35$ and a flux of $1.99_{-0.51}^{+0.55} \times 10^{-11} \mathrm{erg} \mathrm{cm}^{-2} \mathrm{~s}^{-1}$.

\section{NUSTAR DATA : ANALYSIS AND RESULTS}

NuSTAR (Harrison et al. 2013) observed 4C 50.11 with its focal plane module A (FPMA) and B (FPMB) X-ray telescopes, on 2015 December 14 for an exposure time of $20.5 \mathrm{ks}$ (Observation ID: 60160177002). We processed the data with the NuSTAR Data Analysis Software NUSTARDAS version 1.3.1. We cleaned and calibrated the unfiltered event files using standard filtering criteria with the NUPIPELINE task and $N u S T A R$ CALDB version 20151008. The source and background regions were taken from a circular region of radius $70^{\prime \prime}$, and we generated the spectra, response matrices, and ancillary response files, using NUPRODUCTS for both focal plane modules (FPMA and FPMB). The NuSTAR FPMA and FPMB spectra were grouped with a minimum of 20 counts per bin using HEASOFT task GRPPHA. We did not combine the spectra from FPMA and FPMB; instead we jointly fit the two spectra. 
Table 3. Observation Log of Swift XRT observations of 4C 50.11 and the fitting results using a PL model with $N_{\mathrm{H}}$ fixed to the Galactic absorption

\begin{tabular}{|c|c|c|c|c|c|}
\hline ObsID & Date & $\begin{array}{l}\text { Exposure time } \\
(\mathrm{s})\end{array}$ & $\overline{\Gamma_{\mathrm{X}}}$ & $\begin{array}{c}0.3-10 \mathrm{keV} \text { Flux } \\
\left(\times 10^{-12} \mathrm{erg} \mathrm{cm}^{-2} \mathrm{~s}^{-1}\right)\end{array}$ & $\overline{\chi^{2} / \text { d.o.f }}$ \\
\hline 00030879001 & 2007 Jan 25 & 3505 & $0.76_{-0.44}^{+0.44}$ & $5.36_{-1.23}^{+1.75}$ & $\overline{7.6 / 7(\mathrm{C})}$ \\
\hline 00030879002 & 2007 Jan 30 & 1513 & $1.46_{-0.47}^{+0.47}$ & $6.65_{-1.24}^{+1.42}$ & $5.6 / 12(\mathrm{C})$ \\
\hline 00030879003 & 2007 Feb 07 & 3845 & $1.00_{-0.28}^{+0.28}$ & $8.06_{-1.14}^{+1.27}$ & $3.0 / 8$ \\
\hline 00030879004 & 2007 Feb 13 & 5329 & $1.17_{-0.27}^{+0.27}$ & $6.38_{-0.81}^{+0.86}$ & $3.2 / 8$ \\
\hline 00030879005 & 2007 Feb 15 & 5276 & $1.16_{-0.24}^{+0.24}$ & $6.48_{-0.74}^{+0.78}$ & $9.0 / 11$ \\
\hline 00036308001 & 2007 Nov 05 & 8778 & $1.20_{-0.13}^{+0.13}$ & $8.47_{-0.62}^{+0.63}$ & $34.0 / 30$ \\
\hline 00036308002 & 2007 Dec 02 & 11952 & $1.05_{-0.11}^{+0.11}$ & $9.02_{-0.59}^{+0.61}$ & $49.6 / 40$ \\
\hline 00036308003 & 2008 Aug 13 & 2612 & $0.83_{-0.36}^{+0.35}$ & $7.99_{-1.43}^{+1.94}$ & $11.1 / 11(\mathrm{C})$ \\
\hline 00036308004 & 2008 Oct 23 & 2173 & $1.17_{-0.51}^{+0.52}$ & $7.02_{-1.37}^{+2.05}$ & $14.4 / 7(\mathrm{C})$ \\
\hline 00036308005 & 2008 Oct 24 & 9514 & $1.10_{-0.14}^{+0.14}$ & $7.10_{-0.59}^{+0.61}$ & $18.7 / 23$ \\
\hline 00036308006 & 2008 Oct 27 & 873 & $0.80_{-0.71}^{+0.71}$ & $8.79_{-2.30}^{+3.59}$ & $3.3 / 7(\mathrm{C})$ \\
\hline 00030879006 & 2010 Apr 10 & 3780 & $0.82_{-0.29}^{+0.29}$ & $6.70_{-1.09}^{+1.40}$ & $6.1 / 15(\mathrm{C})$ \\
\hline 00030879007 & 2010 Nov 01 & 3930 & $0.96_{-0.36}^{+0.36}$ & $6.06_{-1.01}^{+1.36}$ & $8.5 / 11(\mathrm{C})$ \\
\hline 00036308007 & 2014 Feb 02 & 3854 & $1.14_{-0.23}^{+0.23}$ & $8.27_{-0.99}^{+1.04}$ & $9.3 / 10$ \\
\hline 00080948001 & 2015 Dec 14 & 5011 & $1.25_{-0.17}^{+0.17}$ & $9.08_{-0.85}^{+0.87}$ & $27.5 / 17$ \\
\hline
\end{tabular}

Note-(1) Observation ID used for the analysis; (2) date of observation; (3) exposure time in seconds for each observation; (4) photon index; (5) unabsorbed flux in $0.3-10 \mathrm{keV}$ band derived using cflux model; (6) the $\chi^{2} /$ d.o.f value for the model, where C-statistics is indicated by $\mathrm{C}$.

The NuSTAR spectra in the 3-79 keV energy range were fitted with an absorbed PL model, where the absorption was fixed at the Galactic value. The fit yielded photon index $\Gamma_{\mathrm{X}}=1.52 \pm 0.04$, an unabsorbed flux (derived using cflux model) of $(3.05 \pm 0.14) \times$ $10^{-11} \mathrm{erg} \mathrm{cm}^{-2} \mathrm{~s}^{-1}$, with $\chi^{2} /$ d.o.f $=342.8 / 390$. The photon index obtained with the absorbed PL model is the same as $\Gamma_{2}$ obtained from the broken PL model for the joint NuSTAR and Swift XRT spectra in the $0.3-79$ $\mathrm{keV}$ energy range (see below $§ 4.1$ ).

\subsection{Joint NuSTAR and Swift XRT Analysis}

The simultaneous observations of $4 \mathrm{C} 50.11$ with $\mathrm{NuS}$ $T A R$ and Swift XRT were performed on 2015 December 14. We thus studied the X-ray spectrum of $4 \mathrm{C} 50.11$ over the wide energy range of $0.3-79 \mathrm{keV}$. The broad-band spectrum was fitted with a PL, a PL plus extra absorption component, and a broken PL. In all models, the absorption component (tbabs) was fixed at the Galactic value. The best-fit spectral parameters obtained from the simultaneous fitting are given in Table 4 . The crosscalibration uncertainties between the three telescopes (NuSTAR FPMA, FPMB, and Swift XRT) were considered by adding a multiplicative constant in the model, which was frozen at 1 for the FPMA spectrum and free to vary for the FPMB and XRT spectra. The PL model provided an acceptable fit for the joint spectrum with $\Gamma_{\mathrm{X}}=1.51_{-0.03}^{+0.04}$ and $\chi^{2} /$ d.o.f $=373.1 / 407$, while the addition of an extra absorption at the redshift of the source improved the fit by $\Delta \chi^{2} \sim 9$ for the loss of one extra degree of freedom at a confidence of $>99.5 \%$. The extra absorption column density obtained by this fit is $5.47_{-3.10}^{+3.50} \times 10^{22} \mathrm{~cm}^{-2}$. The broken PL model fur-

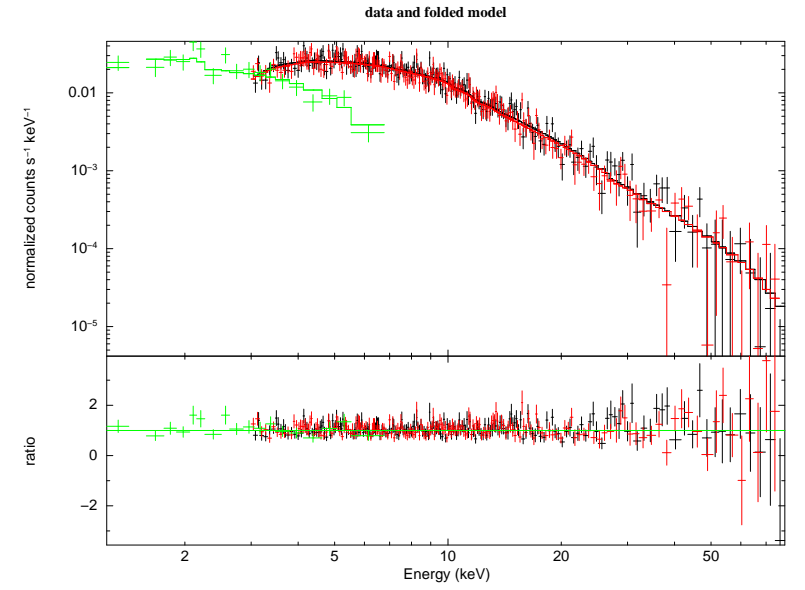

Figure 7. NuSTAR (red and black points) and Swift-XRT (green points) spectra and residuals of $4 \mathrm{C} 50.11$, simultaneously fitted with a broken PL.

ther improved the spectral fit compared to the PL plus the extra absorption model. The spectrum and model fit are shown in Figure 7. The difference in the crosscalibration between FPMA and FPMB was $<4 \%$ in all models, while for the XRT spectrum it was slightly larger but always less than $13 \%$. This difference became larger $(\sim 23 \%)$ when a single PL model was used.

\section{DISCUSSION}

\subsection{Gamma-ray Properties}

We have studied the $\gamma$-ray properties of $4 \mathrm{C} 50.11$ by analyzing the Fermi-LAT data, and confirmed the prediction in Acosta-Pulido et al. (2010) that 4C 50.11 is 
Table 4. Simultaneous fit of NuSTAR and Swift XRT data

\begin{tabular}{lll}
\hline \hline Model & Parameter & Value \\
\hline $\mathrm{PL}$ & $\Gamma_{\mathrm{X}}$ & $1.51_{-0.03}^{+0.04}$ \\
& Flux $(0.3-79 \mathrm{keV})$ & $3.28_{-0.13}^{+0.14}$ \\
& $\chi^{2} /$ d.o.f & $373.1 / 407$ \\
\hline $\mathrm{PL}+$ & $\Gamma_{\mathrm{X}}$ & $1.56 \pm 0.05$ \\
Extra absorber & $\mathrm{N}_{\mathrm{H}}^{z}\left(\times 10^{22} \mathrm{~cm}^{-2}\right)$ & $5.47_{-3.10}^{+3.50}$ \\
& Flux $(0.3-79 \mathrm{keV})$ & $3.21 \pm 0.14$ \\
& $\chi^{2} /$ d.o.f & $363.9 / 406$ \\
\hline Broken PL & $\Gamma_{1}$ & $0.05_{-1.71}^{+0.88}$ \\
& $E_{\text {break }}$ & $2.13_{-0.31}^{+0.62}$ \\
& $\Gamma_{2}$ & $1.52_{-0.03}^{+0.04}$ \\
& Flux $(0.3-79 \mathrm{keV})$ & $3.23_{-0.13}^{+0.14}$ \\
& $\chi^{2} /$ d.o.f & $360.5 / 405$ \\
\hline
\end{tabular}

Note-The errors are at the $90 \%$ confidence level. Flux is in unit of $10^{-11} \mathrm{erg} \mathrm{cm}^{-2} \mathrm{~s}^{-1}$ and corrected for the absorption.

a luminous $\gamma$-ray emitter. From the temporal analysis, we found that the $\gamma$-ray flux during the active period increased by $>3$ times compared to the quiescence level. Moreover, two distinct $\gamma$-ray flares were well seen in the $0.1-1 \mathrm{GeV}$ light curve during this period. Fitting the source emission with a PL, we found that the photon index is possibly inversely proportional to flux (see Figure 2), i.e., the source became harder when brighter. This relationship was also found when the data in the large time intervals (i.e., P1-P6) were studied, as shown in Figure 5.

Our temporal analysis has shown that 4C 50.11 exhibited variability on the time scale of several hours, which is not commonly seen for high redshift blazars. A blazar jet is produced at the central region around the super-massive black hole $(\mathrm{SMBH})$, and as the inner region can not be resolved with current telescopes (note that thus far, M87 is the only source resolved with the current observing facilities, which reaches several Schwarzschild radius, see Hada et al. 2011), variability is a useful feature for probing this region. Given the variability timescale of $4 \mathrm{C} 50.11$, the causality implies that the size of the emission region is $R=$ $t_{v a r} c \delta /(1+z)=4.2 \times 10^{14}(\delta / 10)\left(t_{v a r} / 1 \mathrm{~h}\right) \mathrm{cm}$. The central BH mass of this source is $\approx 4.68 \times 10^{9} \mathrm{M}_{\odot}$ and corresponding Schwarzschild radius is $1.382 \times 10^{15}$ cm (Acosta-Pulido et al. 2010). Therefore the dominant emission region should be only a small part of the whole jet. Assuming the low-energy X-ray emission is produced from the same region, the $\gamma$-ray photons could be absorbed by X-ray photons through the pair production effect. The strength of this absorption is mainly dependent on the X-ray energy density, which will decrease if emission is relativistic Doppler beamed. Therefore, the observed $\gamma$-ray and X-ray data can be used to constrain the jet Doppler factor. Because the $\gamma$-ray photons actually escape from the emission region, the several-hours timescale constrains the lower limit of the beaming factor, $\delta \gtrsim$ $11.8\left[\left(1 \mathrm{~h} / t_{\mathrm{var}}\right)\left(1 \mathrm{keV} / \epsilon_{X}\right)\left(L_{\epsilon_{X}} / 10^{46} \mathrm{erg} \mathrm{s}^{-1}\right)\right]^{1 / 4}$, where $\epsilon_{X} E_{\gamma}=20.61(\delta / 10)^{2}$. From the Fermi-LAT observation, the highest probability of $>10 \mathrm{GeV}$ photons from the source was $87 \%$ of the $\sim 10.3 \mathrm{GeV}$ photon (see Table $1 ; \sim 26.5 \mathrm{GeV}$ in the AGN frame). Considering the $\mathrm{X}$-ray luminosity of the source obtained in this study, the Doppler beaming factor $\delta \gtrsim 8.1\left(t_{v a r} \sim 4 \mathrm{~h}\right)$. The value indicates again that the jet is highly relativistic and has a small viewing angle with respect to our line of sight, which is consistent with the fact that the apparent superluminal motion reaches $\beta_{a p p} \sim 6.3 \pm 1.1$ and the central jet changes the direction about $\sim 100^{\circ}$ (Molina et al. 2014; Agudo et al. 2007).

The $\gamma$-ray spectra show curvature, and a simple PL is not the most favored model (especially for fitting the emission at the high-energy end). Many blazars have shown similar break/curvature properties, which are generally explained as due to the absorption by emission from the BLR or intrinsic curvature. For example, the blazar 3C 454.3 shows a significant break at 2-3 $\mathrm{GeV}$, and the break is considered to be caused by the absorption by BLR photons (Finke \& Dermer 2010). On the other hand, Cerruti et al. (2013) presented detailed SED modeling of 3C 454.3 and found that the break can be well reproduced if the electron energy distribution is log-parabolic, which implies that the break is intrinsic. The detection of $>10 \mathrm{GeV}$ photons from 4C 50.11, which can be easily absorbed by its BLR photons if they are produced within the BLR (Liu \& Bai 2006), suggests that the absorption effect is not the reason for the curved spectra seen from the source. Chen (2014) found that both synchrotron and IC components of blazars can be well fitted by a log-parabola function for a large sample of blazars. Taking the coefficient of the second-order term of the log-parabola as a surrogate of curvature, a significant correlation between curvature and peak energy is a natural result due to the stochastic acceleration mechanism. This correlation has been widely reported through broadband SED fitting (Chen 2014; Xue et al. 2016) or seen in X-ray spectra of a single source (Massaro et al. 2004a,b, 2008). For the first time, we found such a correlation in the $\gamma$-ray spectra of a blazar. The feature also suggests that the $\gamma$-ray break/curvature in 4C 50.11 is likely intrinsic.

\subsection{X-ray Properties}

We have investigated the X-ray properties of 4C 50.11 using the Swift XRT and NuSTAR observations. The source showed variability in the long-term Swift XRT light curve. While its intensity was at the high end of the variation range during the $\gamma$-ray flaring period (Figure 6 ; only the 2014 February Swift observation was conducted in the time period), no significant correlated activity was seen. The X-ray spectral parameters obtained in the active period did not have drastical changes either. As discussed below, we considered that X-rays and $\gamma$-rays are produced from the IC scattering radiation by the same electron population. Because the cooling timescale 
of electrons in the lower energy part (emit the X-rays) is larger than the timescale of higher energy part (emit the $\gamma$-rays), one can expect that the X-ray variability timescale would be larger than that of the $\gamma$-rays.

Using a simple PL to describe the spectra, it is clear that the source displayed a rather hard and relatively stable photon index $\left(\Gamma_{X}=0.8-1.5\right)$. Despite the large uncertainties of the photon index, a marginal hint may exist for that spectra were harder when the source became brighter. At $\gamma$-rays, a similar trend may also be seen (Figure $2 \& 5$ ), suggesting that $\gamma$-ray and X-ray emission was possibly produced from the same electron population.

The spectral steepening of the soft X-ray spectrum has been widely found in high-redshift radio loud quasars (e.g. Yuan et al. 2006, and references therein). The steepening may be due to either the intrinsic absorption with column densities of the order of $10^{22}-10^{23} \mathrm{~cm}^{-2}$ or the low energy cut-off in the energy distribution of electron population in the jet (Fabian et al. 2001a,b; Worsley et al. 2004a,b). In the excess absorption scenario, high $\mathrm{N}_{\mathrm{H}}^{z}$ may be the dense plasma in form of a wind or outflow (Fabian 1999). However in the radioloud quasars like 4C 50.11, the relativistic jet along the line-of-sight can remove the gas column efficiently. Moreover in our SED fitting (see §5.3), the X-ray and $\gamma$ ray emission regions are outside the BLR region, which thus does not favor the excess absorption scenario for $4 \mathrm{C} 50.11$.

If there is a low energy cut-off in the energy distribution of the electron population, a spectrum is expected to flatten in the soft energy band (Fabian et al. 2001a; Tavecchio et al. 2007; Sambruna et al. 2007). This scenario requests a broken PL model, where the cut-off in the soft X-ray band can be naturally explained as the intrinsic curvature of the spectrum near the lowenergy end of the IC component. The soft X-ray steepening is then an intrinsic feature of a source. Among the four examined observations of 4C 50.11, the spectra were relatively well described by the broken PL model of $\Gamma_{1} \sim-0.3-+0.9$ below the break energy $E_{\text {break }}=$ $2.1-3.3 \mathrm{keV}$, and $\Gamma_{2} \sim 1.4-1.8$. The simultaneous observations of $4 \mathrm{C} 50.11$ by Swift and NuSTAR showed that the broad-band X-ray spectrum is better modeled by a broken PL than by a PL or a PL plus extra absorption model. We found $\Gamma_{1}=0.05_{-1.71}^{+0.88}$ below the break energy, $E_{\text {break }}=2.13_{-0.31}^{+0.62} \mathrm{keV}$, and $\Gamma_{2}=1.52_{-0.03}^{+0.04}$. In the 14-195 keV energy range of the Swift BAT, the source was found to have photon index $\Gamma_{\mathrm{X}}=1.51 \pm 0.35$ (Baumgartner et al. 2013), which is well in agreement with $\Gamma_{2}$ obtained in our broad-band fit. Thus the steepening is very likely the intrinsic feature of the source.

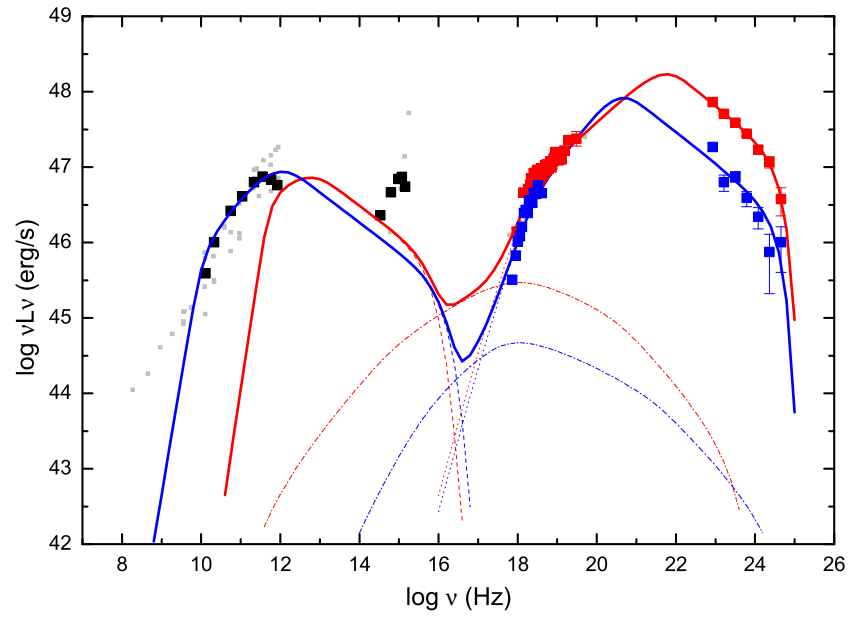

Figure 8. Broad-band SED of $4 \mathrm{C} 50.11$, where the black and grey data points are from Acosta-Pulido et al. (2010) and NED, respectively, and the red and blue squares are the $\mathrm{X}$-ray and $\gamma$-ray spectra in the active and quiescent states, respectively. The solid, dashed, dotted and dot-dashed lines represent the total, synchrotron, external Compton and synchrotron self Compton emission respectively.

We collected the archival radio and optical data for 4C 50.11 from Acosta-Pulido et al. (2010) and NED ${ }^{2}$ respectively. These data were combined with X-ray and $\gamma$-ray data in this work and the broad-band SED of the source is shown in Figure 8. In this SED, emission from the relativistic jet dominated except at optical wavelengths. The optical emission reached a peak luminosity of $\sim 10^{47} \mathrm{erg} \mathrm{s}^{-1}$ and appeared as a significant bump, which should be thermal arising from the central thick accretion disk. As $4 \mathrm{C} 50.11$ hosts a very massive $\mathrm{BH}$ with $M=4.68 \times 10^{9} M_{\odot}$, the thermal disk emission reaches $\sim 30 \%$ of the Eddington limit (Acosta-Pulido et al. 2010). The broad-band SED is not simultaneous, except the two sets of Swift X-ray and the corresponding Fermi $\gamma$-ray data in the active and quiescent states (the red and blue squares, respectively, in Figure 8). Nevertheless, We modeled the broad-band SED by using a standard blazar emission model: one zone synchrotron plus inverse Comptonization model. This model was widely used in blazar SED modeling (e.g., Ghisellini et al. 2010; Chen et al. 2012). The emission region is assumed to be a homogeneous sphere with radius $R$ embedded in the magnetic field $B$. A broken power-law electron energy distribution,

$$
N(\gamma)= \begin{cases}N_{0} \gamma^{-p_{1}} & \gamma_{\min } \leq \gamma \leq \gamma_{0} \\ N_{0} \gamma_{0}^{p_{2}-p_{1}} \gamma^{-p_{2}} & \gamma_{0}<\gamma \leq \gamma_{\max },\end{cases}
$$

was assumed in our calculation. The parameters of this model include the radius $R$ of the blob, the magnetic field strength $B$, electron break energy $\gamma_{0}$, the minimum 
Table 5. SED Model parameters for 4C 50.11

\begin{tabular}{lccccccccc}
\hline \hline State & $\begin{array}{c}B \\
(\mathrm{G})\end{array}$ & $\begin{array}{c}R / 10^{16} \\
(\mathrm{~cm})\end{array}$ & $N_{0}$ & $\delta$ & $\gamma_{0}$ & $\gamma_{\min }$ & $\gamma_{\max }$ & $p_{1}$ & $p_{2}$ \\
\hline Active & 0.5 & 7.5 & 2262 & 29 & 295 & 6 & 8259 & 2.0 & 3.8 \\
Quie. & 0.17 & 3300 & 0.015 & 4 & 546 & 40 & 54600 & 1.5 & 3.8 \\
\hline
\end{tabular}

and maximum energy, $\gamma_{\min }$ and $\gamma_{\max }$, of the electrons, the normalization of the particle number density $N_{0}$, and the indices $p_{1,2}$ of the broken power law particle distribution, the jet Doppler factor (assumed to be equal to the bulk Lorentz factor), and the spectrum of the external seed photons. The frequency and luminosity can be transformed from the jet frame to observational frame as: $\nu=\delta \nu^{\prime} /(1+z)$ and $\nu L_{\nu}=\delta^{4} \nu^{\prime} L_{\nu^{\prime}}^{\prime}$, where the Doppler factor $\delta=1 /[\Gamma(1-\beta \cos \theta)]$, and the prime represents the value measured in the jet frame. The synchrotron self-absorption and the Klein-Nishina effect in the IC scattering were properly considered in our calculations. Both the self-synchrotron Compton (SSC) scattering and external Compton (EC) scattering (external seed photons from the BLR and dusty torus were taken into account) were included in the calculation of the Compton scattering in the blob. As mentioned above, the optical emission is thermal arising from the accretion disk. Therefore, we excluded this component in the modeling. The non-simultaneous radio-optical data were taken as the upper limits throughout the modeling. The model curves are shown in Figure 8. The jet parameters found for the active and quiescent states are given in Table 5. Throughout the fitting, we found that the emission region must be outside the BLR, and the main external seed photons are from the dusty torus. This is consistent with the above estimate based on $\gamma-\gamma$ absorption effect, i.e., the observed high-energy $>10$ $\mathrm{GeV}$ photons are optically thick if produced within the BLR. Having the model parameters, the jet power can be calculated as, $L_{\text {jet }} \simeq \pi R^{2} \beta \Gamma^{2} c U_{\text {tot }}^{\prime}$, where the total energy density measured in the rest frame of the jet, $U_{\text {tot }}^{\prime}=U_{\mathrm{e}}^{\prime}+U_{\mathrm{B}}^{\prime}+U_{\mathrm{p}}^{\prime}$. The energy density for electrons $U_{\mathrm{e}}^{\prime}=m_{\mathrm{e}} c^{2} \int N(\gamma) \gamma d \gamma$, while the proton energy density $U_{\mathrm{p}}^{\prime}=U_{\mathrm{e}}^{\prime}\left(m_{\mathrm{p}} / m_{\mathrm{e}}\right) /\langle\gamma\rangle$ if charge neutrality for pure hydrogen plasma is assumed. The estimated jet power $\sim 2.5 \times 10^{47} \mathrm{erg} \mathrm{s}^{-1}$ for the active state, which is larger than the disk luminosity $\left(1.8 \times 10^{47} \mathrm{erg} \mathrm{s}^{-1}\right)$, and reaches $\sim 42 \%$ of the Eddington power. The estimated magnetic field is $\sim 0.53 \mathrm{G}$, which is roughly consistent with the value estimated from the radio technique $\sim 0.7$ G (Agudo et al. 2007).

\section{SUMMARY}

We have studied the $\gamma$-ray and X-ray properties of the high redshift blazar 4C 50.11 from analyzing the FermiLAT, Swift, and NuSTAR data. The main results are summarized as the following.

- From Fermi-LAT monitoring, the source was found to be in an active state since approximately MJD 56482. During the state, the source's $\gamma$-ray flux largely increased almost by one order of magnitude (averaged on 5 days bin) compared to the quiescent level. A correlation between the peak energy and spectral curvature (and between the photon index and flux if a PL model is considered) was seen. This correlation is the first time clearly detected in the $\gamma$-ray spectra of a blazar. Other important properties found are the severalhours variability and the possible detection of several $>10 \mathrm{GeV}$ photons. These properties have helped constrain the physical properties of the jet of the blazar.

- The source showed flux variability in the Swift and NuSTAR data we have analyzed, but no obvious flux enhancement or spectral changes related to the $\gamma$-ray active state were seen. As long as the data quality allows, we have found that a broken power law provided the best fit to the X-ray spectra, with an extremely hard spectrum $(\Gamma \sim 0.1)$ below the break energy. This spectral feature is likely due to the low-energy cutoff in the energy distribution of the photon-emitting electron population. Similar to that seen at $\gamma$-rays, spectra possibly hardened when the source was brighter, suggesting that emission in the both energy bands was produced from the same electron population.

- We have constructed the broad-band SED for 4C 50.11, though not simultaneous, and provided a model fit (one-zone synchrotron plus inverse Comptonization model) to the SED. From modeling, properties of the emission region were derived. We have found that the emission region should be outside the BLR and the jet contained $42 \%$ of the Eddington power during the active state.

\section{ACKNOWLEDGMENTS}

We acknowledge the use of data from Fermi Science Support Center (FSSC), and Swift, NuSTAR data from the High Energy Astrophysics Science Archive Research Center (HEASARC), at NASAs Goddard Space Flight Center. This research has made use of the High Performance Computing Resource in the Core Facility for Advanced Research Computing at Shanghai Astronomical Observatory, the NuSTAR Data Analysis Software (NUSTARDAS) jointly developed by the ASI Science Data Center (ASDC, Italy) and the California Institute of Technology (Caltech, USA). VJ acknowledges the financial support from Chinese Academy of Sciences through President's International Fellowship Initiative (CAS PIFI, Grant No. 2015PM059). This research was supported by the Strategic Priority Research Program "The Emergence of Cosmological Structures" of the Chinese Academy of Sciences (Grant No. XDB09000000) and the National Natural Science Foundation of China (11373055, 11633007). Z.W. acknowledges the support by the CAS/SAFEA International Partnership Program for Creative Research Teams. 


\section{REFERENCES}

Abdo, A. A., Ackermann, M., Ajello, M., et al. 2011, ApJL, 733, 26

Acero, F., Ackermann, M., Ajello, M., et al. 2015, ApJS, 218, 23

Acosta-Pulido, J. A., Agudo, I., Barrena, R., et al. 2010, A\&A, 519,5

Agudo, I., Bach, U., Krichbaum, T. P., et al. 2007, A\&A, 476, 17

Agudo, I., Bach, U., Krichbaum, T. P., et al. 2008, ASPC, 386, 249

Agudo, I., Thum, C., Wiesemeyer, H., \& Krichbaum, T. P. 2010, ApJS, 189, 1

Barthelmy, S. D., Barbier, L. M., Cummings, J. R., et al. 2005, SSRv, 120, 143

Baumgartner, W. H., Tueller, J., Markwardt, C. B., et al. 2013, ApJS, 207, 19

Burrows, D. N., Hill, J. E., Nousek, J. A., et al. 2005, SSRv, 120, 165

Carpenter, B., Ojha, R., D'Ammando, F., et al. 2014, ATel, 5838,1

Cash, W. 1979, ApJ, 228, 939

Cerruti, M., Dermer, C. D., Lott, B., Boisson, C., \& Zech, A. 2013, ApJL, 771, L4

Chen, L. 2014, ApJ, 788, 179

Chen, L., Cao, X., \& Bai, J. M. 2012, ApJ, 748, 119

Fabian, A. C. 1999, MNRAS, 308, L39

Fabian, A. C., Celotti, A., Iwasawa, K., et al. 2001, MNRAS, 323,373

Fabian, A. C., Celotti, A., Iwasawa, K., \& Ghisellini, G. 2001, MNRAS, 324, 628

Fey, A. L., Ma, C., Arias, E. F., et al. 2004, AJ, 127, 3587

Finke, Justin D.; Dermer, Charles D. 2010, ApJ, 714, 303

Foschini, L., 2010, ATel, 2517, 1

Foschini, L., Bonnoli, G., Ghisellini, G., et al. 2013, A\&A, 555, 138
Gehrels, N., Chincarini, G., Giommi, P., et al. 2004, ApJ, 611, 1005

Ghisellini, G., Tavecchio, F., Foschini, L., et al. 2010, MNRAS, 402, 497

Güver, T., Özel, F. 2009, MNRAS, 400, 2050

Hada, K., Doi, A., Kino, M., et al. 2011, Nature, 477, 185

Harrison, F. A., Craig, W. W., Christensen, F. E., et al. 2013, ApJ, 770, 103

Kalberla, P. M. W., Burton, W. B., Hartmann, D., et al. 2005, A\&A, 440, 775

Krauss, F., Carpenter, B., Ojha, R., 2014, ATel, 5878, 1

Liu, H. T., Bai, J. M., 2006, ApJ, 653, 1089

Massaro, E., Perri, M., Giommi, P., \& Nesci, R. 2004, A\&A, 413, 489

Massaro, E., Perri, M., Giommi, P., Nesci, R., \& Verrecchia, F. 2004, A\&A, 422, 103

Massaro, F., Tramacere, A., Cavaliere, A., Perri, M., \& Giommi, P. 2008, A\&A, 478, 395

Molina, S., Agudo, I., Gómez, J., et al. 2014, A\&A, 566, 26

Pauliny-Toth, I. I. K., Wade, C. M., Heeschen, D. S., 1966, ApJS, 13, 65

Sambruna, R. M., Tavecchio, F., Ghisellini, G., et al. 2007, ApJ, 669,884

Tavecchio, F., Maraschi, L., Ghisellini, G., et al. 2007, ApJ, 665, 980

Urry, C. M. and Padovani, P. 1995, PASP, 107, 803

Wilms, J., Allen, A., \& McCray, R. 2000, ApJ, 542, 914

Worsley, M. A., Fabian, A. C., Celotti, A., \& Iwasawa, K. 2004, MNRAS, 350, L67

Worsley, M. A., Fabian, A. C., Turner, A. K., Celotti, A., \& Iwasawa, K. 2004, MNRAS, 350, 207

Xue, R., Luo, D., Du, L. M., et al. 2016, arXiv:1609.05697

Yuan, W., Fabian, A. C., Worsley, M. A., \& McMahon, R. G. 2006, MNRAS, 368, 985 